NOTICE: this is the author's version of a work that was accepted for publication in Ecological Modelling. Changes resulting from the publishing process, such as peer review, editing, corrections, structural formatting, and other quality control mechanisms may not be reflected in this document. Changes may have been made to this work since it was submitted for publication. A definitive version was subsequently published in Ecological Modelling, vol. 221, no. 19, 2010, http://dx.doi.org/10.1016/j.ecolmodel.2010.04.018 


\title{
Comparison of alternative strategies for invasive species distribution modeling
}

\author{
Todd P. Robinson ${ }^{1 *}$, Rieks D. van Klinken ${ }^{2}$, Graciela Metternicht ${ }^{3}$. \\ ${ }^{1}$ Department of Spatial Science, Curtin University of Technology, GPO Box U1987, \\ Perth, WA 6845, Australia \\ ${ }^{2}$ CSIRO Entomology, 120 Meiers Rd, Indooroopilly, Queensland, 4068, Australia, \\ ${ }^{3}$ United Nations Environment Programme, Panama City, Panama
}

\begin{abstract}
Species distribution models (SDMs) can provide useful information for managing biological invasions, such as identification of priority areas for early detection or for determining containment boundaries. However, prediction of invasive species using SDMs can be challenging because they typically violate the core assumption of being at equilibrium with their environment, which may lead to poorly guided management resulting from high levels of omission. Our goal was to provide a suite of potential decision strategies (DSs) that weren't reliant on the equilibrium assumption but rather could be chosen to better match the management application, which in this case was to ensure containment through adequate surveillance. We used presence-only data and expert knowledge for model calibration and presence/absence data to evaluate the potential distribution of an introduced mesquite (Leguminoseae: Prosopis) invasion located in the Pilbara Region of northwest Western Australia. Five different DSs with varying levels of conservatism/risk were applied to a weighted linear combination (WLC) model using ordered weighted averaging. The performance of DSs over all possible thresholds was examined using receiver operating characteristic (ROC) analysis. DSs not on the convex hull of the ROC curves were discarded. Two threshold determination methods (TDMs) were compared on the two remaining DSs, one that assumed equilibrium (by maximizing overall prediction success) and another that assumed the invasion was ongoing (using a 95\% threshold for true positives). The most conservative DS fitted the validation data most closely but could only predict $75 \%$ of the presence data. A more risk-taking DS could predict $95 \%$ of the presence data, which identified 8.5 times more area for surveillance, and better highlighted known populations that are still rapidly invading. This DS and TDM coupling was considered to be the most appropriate for our management application. Our results show that predictive niche modeling was highly sensitive to risk levels, but that these can be tailored to match specified management objectives. The methods implemented can be readily adapted to other invasive species or for conservation purposes.
\end{abstract}

\section{Key-words}

Biological Invasions, mesquite, multi-criteria evaluation, ordered weighted averaging, ROC, risk

\footnotetext{
${ }^{*}$ Correspondence: Todd P. Robinson, Department of Spatial Science, Curtin University of Technology, GPO Box U1987, Perth, WA 6845, Australia. E-mail: pfrass@bigpond.com.au; Tel/Fax: +61 089454 2007
} 


\section{Introduction}

Species distribution models generally proceed by first identifying environmental characteristics that are associated with a species occurrence and then extrapolating this information to detect other areas that possess similar characteristics (Underwood et al., 2004). For invasive species, this information can then be used to develop management strategies, determine containment boundaries and identify priority areas for early detection and rapid response (Elith et al., 2006; Morisette et al., 2006; Jiménez-Valverde and Lobo, 2007). However, such predictions can be challenging for introduced invasive species, which frequently have wide ecological niches and may not yet have reached equilibrium within their new environment (Sutherst and Bourne, 2009; van Klinken et al. 2009; Vávlavík and Meentemeyer, 2009). Hence, the key challenge for predicting the potential ranges of an invasive species is handling the uncertainty inherent in distributional data where it has not yet reached the full extent of habitat that could support it. How this uncertainty is handled will depend on management objectives (e.g. eradication, containment or impact reduction) and on the resources available to implement the management strategy. In this paper we explicitly consider the relationship between uncertainty (considered within a risk framework) and management. In this approach, the model assuming equilibrium becomes just one of a suite of possible scenarios that are assessed to suit management objectives (Underwood et al., 2004; Jiménez-Valverde et al., 2008; Sutherst and Bourne, 2009).

A number of techniques for species distribution modeling have been reviewed in the literature (c.f. Franklin, 1995; Guisan and Zimmermann, 2000). Correlative models, for example, rely on the detection of a correlation between species distribution records and the environmental predictor variables used to make predictions (Beerling et al., 1995; Robertson et al., 2003). However, particularly with introduced species, there may not be an overt cause for the correlation (Beerling et al., 1995). In addition, correlative models assume distribution records represent the entire range of sites that can be occupied by the target species (the equilibrium assumption) and thus, when used with accurate presence and absence records, approximates the actual or realized distribution (Jiménez-Valverde et al., 2008). However, the equilibrium assumption is violated by actively expanding invasive species and, therefore, the actual distribution relating to a point in time may be a conservative representation (Franklin, 1995; Beerling et al., 1995; Austin, 2002; Hulme, 2003). While a conservative modeling approach is likely to increase the likelihood of predicting sites where a species exists (few false positives), or may survive, it may severely underestimate areas where a species may potentially exist (Jiménez-Valverde et al., 2008) thereby misdirecting management action and policy development. For example, underestimation may result in invasion going unnoticed until the species is well established (Robertson et al., 2004) and beyond successful eradication (Rejmánek and Pitcairn, 2002). Instead, it can be argued that relaxing the level of conservatism (thereby increasing risk levels) to capture a greater number of known presence sites (potentially at the expense of a greater number of false positives) may be more desirable for providing an early warning for species that are still expanding.

Recently, there has been a greater emphasis on identifying modeling approaches (e.g. Sutherst and Bourne, 2009), and alternative techniques (e.g. geographically weighted regression) for species that have not yet reached equilibrium (Austin, 2007). A common approach has been to use profile techniques (e.g. Tsoar et al., 2007), which do not take into account data on a species absence and more commonly tend towards 
approximating areas where a species could live (i.e., the potential distribution) rather than simply where it currently does live (i.e., the actual/realized distribution). These techniques (and others) may also be dichotomized into predicted presence and absence by choosing a suitable threshold that decreases the false negative rate at the expense of an increased false positive rate (e.g. Fielding and Bell, 1997). Our aim is to overcome the limitations of causality and under prediction by using a deductive approach within a geographical information system (GIS) where the rules are derived from a combination of expert knowledge and empirically derived data as described in Eastman et al. (1993); Franklin (1995); and Robertson et al. (2004) and the level of conservatism (or risk) can be adjusted during the process of combining data layers (ecological variables) to suit prediction outcomes. The use of such risk adjusting techniques to develop a range of scenarios (decision strategies) with varying levels of conservatism (or risk) has received very little attention to date. Here we explore its utility in the context of modeling invasive organisms.

This study adopts a standard, moderate risk-taking, multi-criteria evaluation tool (weighted linear combination (WLC)) (Jiang and Eastman, 2000) and couples it with a risk-adjusting technique known as ordered weighted averaging (OWA) to develop a series of alternative decision strategies. We develop five alternative decision strategies (two more conservative than the WLC model, one equal in risk to the WLC model, and two more risk-taking) for identifying the distribution of an invasive mesquite (Leguminoseae: Prosopis spp.) population in the Pilbara Region of northwest Western Australia. The population is currently largely restricted to a single property, but is thought to have the potential to invade more widely. The primary focus of management is therefore containment of the core infestation, and eradication and surveillance outside of the core infestation. Mesquite presence and absence data were obtained from a previous airborne survey of the entire known population (ca. $1,500 \mathrm{~km}^{2}$ ), which was in turn used to predict habitat suitability across $112,649 \mathrm{~km}^{2}$ of the Pilbara region (van Klinken et al. 2007). Receiver operating characteristic (ROC) analysis and two threshold determination methods (Liu et al. 2005) were used to determine which models would best identify suitable habitat assuming that the population was at equilibrium, and assuming the population was still invading, respectively. Comparison of the alternative outputs is examined in the context of our management goal of identifying areas for early warning and surveillance.

\section{Methods}

\subsection{Target Species}

Several mesquite species (together referred to as "mesquite") are recognized as being highly invasive, both in their native and introduced ranges (Archer, 1995; van Klinken et al., 2006). They are leguminous shrubs or trees that can form dense thorn-forests, resulting in serious economic, environmental and social costs (Hennessy et al., 1983; Gibbens et al., 1992; Goslee et al., 2003). Mesquite reproduces from seeds and typically has a high fecundity, producing one main crop per year. In Australia, it is ranked nationally as one of the twenty most significant weeds (Thorp and Lynch, 2000).

The studied mesquite population is located in the northwest Pilbara region of Western Australia (centred on $21^{\circ} 11^{\prime} 18^{\prime}$ 'S, $115^{\circ} 56^{\prime} 67^{\prime}$ ' $\mathrm{E}$ ) and is the result of an intentional introduction to the Mardie Pastoral Station in the 1930s to serve as a drought and fodder plant as well as for shade for livestock (Meadly, 1962). It has since invaded 
over 150,000 ha, principally on the delta of the Fortescue River where it was first introduced and where most of the approximately 32,500 ha of dense mesquite occurs. However, it is also spreading and increasing in density on adjacent catchments, especially in the Robe River delta to the southwest (van Klinken et al., 2007). The population is described as a hybrid swarm of $P$. pallida, $P$. velutina and $P$. glandulosa var glandulosa (van Klinken and Campbell, 2009). P. pallida belongs to the $P$. juliflora- $P$. pallida complex, which is native to southern Central America, while $P$. velutina and $P$. glandulosa are a complex native to the USA and Mexico (Pasiecznik et al., 2001). The hybridization of these species obtained from diverse geographical areas in the northern hemisphere makes it difficult to extrapolate species-specific observations derived from native range populations to novel environments as has been done in other studies (e.g. De Meyer et al., 2007; Mgidi et al., 2007; Beaumont et al., 2009).

\subsection{Validation and calibration data}

The entire known mesquite infestation was mapped as an 18.5 ha grid-matrix during a visual aerial survey in 2004 (van Klinken et al., 2007). Mesquite was mapped as absent, isolated, moderate or dense, which we converted to categorical presence/absence data. To minimize the effects of spatial dependency we randomly sampled 500 presence data and 500 absence data for model validation. This also avoided the potential bias caused by different levels of prevalence in presence/absence datasets (Manel et al., 2000). An additional randomly sampled partition of 500 presence data was also used as calibration data for standardizing the compound topographic index (see Section 2.3.3). Calibration of the other criteria used in the model from this data was not possible as the aerial survey did not record data for some of the classes that were located beyond the boundary of the current infestation. These criteria were standardized using expert opinion.

\subsection{Criteria selection}

Criteria (environmental variables) were selected based on previous ground-based and remote sensing-based studies (van Klinken et al., 2006; van Klinken et al., 2007; Robinson et al., 2008) designed to determine the habitat preferences of the mesquite population under study. Three criteria were chosen that we considered most influential on the current distribution and were available as GIS layers.

\subsubsection{Pastoral potential}

Pastoral potential, calculated as the number of hectares required to sustain the nutritional requirements of a unit of cattle (carrying capacity), has been mapped into five categories (very high, high, moderate, low and very low) according to the land system types across the Pilbara Region (Payne and Mitchell, 2002). These land systems were mapped on the basis of their distinctive patterns of landforms, soils and vegetation types. The overall pastoral potential for a given land system is derived from a weighted average of its component pastures (van Vreeswyk et al., 2004). For example, pasture types within a land system that are considered to have a low carrying capacity will downgrade that land systems pastoral potential. The 2004 aerial survey (Section 2.2) revealed a strong positive correlation between mesquite density and pasture potential (van Klinken et al., 2007). 


\subsubsection{Land use}

The introduction of livestock is considered to be responsible for the rapid spread of mesquite within its native range from riparian zones, where it had been previously restricted, into upland habitats as seed pods are actively sought after for feed, and excreted with enhanced germination capacity (Brown and Archer, 1987). Cattle are generally considered the most effective herbivores for distributing mesquite since a large proportion of seeds (ca. 60\%; Brown and Archer, 1987) survive through their digestive tract. Hence, the potential distribution of mesquite is dependent, in part, on the presence of cattle.

\subsubsection{Soil moisture}

In a retrospective study using archival aerial photographs it was determined that rates of mesquite colonization were highest in the most mesic (wettest) parts of the landscape (Robinson et al., 2008). Therefore, a steady state wetness index (the compound topographic index (CTI)) was derived from a digital elevation model to act as a surrogate for soil moisture, using Eq. 1 (Gessler et al., 1995):

$$
\mathrm{CTI}=\ln (\alpha / \tan (\beta))
$$

where $\alpha=$ upstream area calculated as (flow accumulation +1$) *\left(\right.$ pixel area in $\mathrm{m}^{2}$ ) and $\beta=$ slope expressed in radians. Flow accumulation is calculated from a flow direction grid, which determines the water flow as the direction of steepest descent. Flow accumulation then records the number of cells that drain into an individual cell in the grid and is thus measured in units of grid cells (Olivera et al., 2002).

\subsection{Multi-criteria evaluation (MCE) model}

The MCE model was constructed using a weighted linear combination with criteria standardized to a common numerical range (Section 2.4.1). Weights were assigned using the analytic hierarchy process (AHP) (Section 2.4.2) (Saaty, 1987; Malczewski, 1999).

\subsubsection{Standardization}

Categorical layers (pastoral potential and land use) were standardized using a ranking procedure, whereby each class is inversely ranked (the least important class $=1$, next least important $=2$, etc.) based on its perceived importance for facilitating mesquite invasion. Once the ranking was established, standardization was based on the rank exponent method as described by Malczewski (1999). In our application we squared the rank position and divided it by the value of the square of the most important class to derive a standardized score from 0 to 1 . The process of squaring the rank position produces a function that decays rapidly, thereby assigning a higher standardized value to the more important classes relative to the lesser important classes.

The five categories of pastoral potential were ranked in sequential order with very high pastoral potential ranked first and very low pastoral potential being ranked fifth (Table 1). Land uses were ranked according to their likelihood for promoting mesquite invasion, with the presence of cattle receiving the highest rank, based on the knowledge that mesquite populations can be dispersal-limited in their absence. All other land uses were ranked equally, resulting in a standardized score of 0.25 (Table 
1), to account for the presence of the more ubiquitous but less effective vectors such as wallaroos and emus.

The continuous compound topographic index (CTI) layer was standardized using a fuzzy membership function (FMF). FMFs assign a value between 0 and 1 to each pixel allowing the transition between non-membership (0) and complete membership (1) to be both continuous and gradual (Zadeh, 1965; Robertson et al., 2004). The shape of the FMF is governed by a set of control points which can either be defined based on expert judgment or determined from calibration data. To construct the FMF for the CTI layer a frequency histogram of the CTI values was constructed using the calibration dataset (see Section 2.2). A right skewed distribution was found, with a minimum of 5.5, and a median of 9.4. Following Burrough and McDonnell (1998) and Robertson et al. (2004) we applied a monotonically increasing s-shaped function, where the minimum marked the lower control point at which the membership function began to rise above 0 , and the median marked the upper control point where all values greater than or equal to it were assigned a fuzzy membership value of 1 (Fig. 1).

\section{<approximate location of Fig. 1>}

\subsubsection{Criteria Weights}

Criteria weights represent the influence of each criterion in the model on the distribution of mesquite. The analytic hierarchy process (AHP) is one method of producing criteria weights. The AHP requires the creation of a reciprocal pair-wise comparison matrix. Entries into the matrix are found from comparison between each layer based on a 9-point rating scale as developed by Saaty (1977) where a value of 1 is given to imply the criteria under comparison are of equal importance to the final solution and 9 expresses extreme importance of one criterion over another. Values in between are used for expressing moderate importance of one criterion over another (3), strong importance (5) and very strong importance (7). If the criteria being compared are deemed to be closer than indicated by this scale, one can use values in between. For example, if one criterion is only slightly more important than another it can be indicated by a value of 2 . Comparisons are made by comparing the row criterion to the column criterion. If the row criterion is of less importance to the column criterion the reciprocal is used (e.g., very strongly less important would be expressed as 1/7). By definition the diagonal entries are all equal to 1 (criteria are equally important when compared to themselves) and the rating in any position $i, j$ will be the reciprocal of that in position $j, i$ (Lippitt et al., 2008). The Principal Eigenvector of this matrix yields the weights applicable to each layer (Saaty, 1987; Malczewski, 1999; Lippitt et al, 2008). A consistency ratio (CR) can be computed from the matrix to express the degree to which pair-wise comparisons form a consistent set of relationships. A matrix with a CR $>0.1$ is considered inconsistent (Saaty, 1977).

The pair-wise comparison matrix and derived weights used in this study are shown in Table 2. Soil moisture (CTI) was considered slightly more important than pastoral potential based on the findings of Robinson et al. (2008) that showed that mesquite colonized and increased significantly more rapidly in the riparian zone than over the uplands, even though both had the same soil type (red loamy soils). Soil moisture was deemed to be moderately more important than land use because, although mesquite is successfully dispersed via livestock, with poor soil moisture it is less 
likely to survive. Pastoral potential was deemed to be slightly more important than land use because while seeds may be widely dispersed by livestock, they are less likely to survive if the environment is poor.

\subsection{Ordered weighted averaging (OWA)}

OWA provides a tool for generating a wide range of decision strategies in decision strategy space (Fig. 2) by applying a set of order weights to criteria that are ranked in ascending order on a pixel-by-pixel basis. The number of order weights is equal to the number of criteria and must sum to one. The position of a set of order weights can be identified in a decision strategy space based on the concepts of trade-off and risk (Yager, 1988; Jiang and Eastman, 2000). Trade-off indicates the degree to which a low standardized value on one layer can be compensated for by a high standardized value on other criteria under consideration. Risk refers to how much each criterion affects the final solution (Jiang and Eastman, 2000; Malczewski, 2006). For example, the most conservative set of order weights are given as $[1,0,0]$ (Fig. 2) for three criteria, which assigns full importance to the lowest pixel value over three criteria, thus all coincident pixels of the three criteria need to be close to 1 to receive a high suitability rating. However, if the first ranked criterion has a low pixel value, despite high coincident pixel values on other criteria, that pixel will be classified as relatively unsuitable.

\section{<approximate location of Fig. 2>}

We chose to manipulate the level of risk and trade-off of the MCE model using five different sets of order weights enabling the creation of five alternative decision strategies ranging from risk-averse (Minimum Risk with order weights $=[1,0,0]$ ), to highly risk-taking (Maximum Risk [0,0,1]) (Fig. 2). The five different decision strategies can be defined by coefficients of risk and trade-off ranging from 0 to 1 , where 0 indicates no risk and no trade-off (Minimum Risk; Fig. 2), and 1 indicates maximum risk (Maximum Risk; Fig. 2) and trade-off (Moderate Risk; Fig. 2) (Malczewski, 1999).

\subsection{Validation}

Predictions from the five alternative decision strategies were assessed using receiver operating characteristic (ROC) analysis based on our validation dataset. For each strategy the favorability score (from 0 to 1 ) was extracted for both the presence and absence data and sorted in ascending order. Thresholds were defined as half the distance between each successive pair. At each threshold the true positive rate (TPR) and the false positive rate (FPR) was calculated using Eqs. 2 and 3, respectively (Fielding and Bell, 1997; Fawcett, 2006).

$$
\begin{aligned}
& T P R=\frac{n P P_{i}}{n P P} \\
& F P R=\frac{n A P_{i}}{n A P}
\end{aligned}
$$


where $n P P_{i}$ is the cumulative number of presence points at threshold $i, n P P$ is the total number of presence points (500), $n A P_{i}$ is the cumulative number of absence points at threshold $i$ and $n A P$ is the total number of absence points (500).

ROC curves were constructed by plotting the coordinates of the TPR (y-axis) and the FPR (x-axis) for all thresholds, for each of the five decision strategies. The trapezoidal rule (Pontius and Schneider, 2001) was used to generate the area under the curve (AUC) statistic, which is a commonly used summary statistic used to indicate model performance. A model that perfectly discriminates between presence and absence records has an AUC of 1 (i.e., perfect discrimination between presence and absence records), while a model predicting mesquite presence and absences no better than by chance has an AUC of 0.5 (Fielding and Bell, 1997; Ayalew and Yamagishi, 2005; Fawcett, 2006).

\subsubsection{Selecting decision strategies}

It is common practice to choose between alternative decision strategies and model types based on the magnitude of the AUC (Zweig and Campbell, 1993). However, as the AUC summarizes performance over all thresholds it is possible for a particular strategy with the highest AUC to be inferior to another in a specific region of interest in ROC space (Fawcett, 2006; Lobo et al., 2008). Therefore, the AUC was only used to identify the decision strategy with the best average performance. Instead, identification of potentially optimal decision strategies was accomplished by identifying those that lie on the convex hull of the set of points in ROC space (Fawcett, 2006). ROC curves that lie on the ROC convex hull have more efficient false positive and true positive rates than those that lie beneath them and thus, these sub-optimal decision strategies can be readily discarded, as was done in this study. Determining which of the remaining decision strategies is the most optimal is dependent on the perceived cost of false negatives (errors of omission) and false positives (errors of commission). Two threshold determination methods (Liu et al., 2005) were applied to the potentially optimal decision strategies on the ROC convex hull to explore the impact of these costs in more detail.

\subsubsection{Threshold determination methods}

The first threshold determination method (TDM) was used to identify the decision strategy that maximized overall prediction success (OPS). This was achieved using the maximum efficiency (ME) statistic, which detects the threshold where the difference between the TPR and the FPR is largest (Lippitt et al., 2008). This threshold was used to dichotomize between the predicted presence and predicted absence of mesquite. The decision strategy with the highest OPS is the one out of all candidates that most accurately matches the validation data and provides a balanced trade-off between false negatives (omission errors) and false positives (commission errors) (Lobo et al., 2008). It is therefore the strategy of choice if the species has invaded all possible niches (i.e., the equilibrium assumption). However, in the process of balancing the omission and commission errors, this method may omit other potential locations, which, in the context of invasive species, is considered a critical limitation for surveillance programs. Therefore, to minimize the exclusion of areas that may still be suitable for mesquite, a subjective, predetermined TDM was implemented to identify the decision strategy that could predict $95 \%$ of known 
mesquite occurrences (i.e., TPR $=95 \%$ ) with the lowest corresponding FPR (Cantor et al., 1999; Liu et al., 2005). In addition to the AUC, FPR and TPR, we also calculated the area predicted by each potentially optimal decision strategy, grouped by TDM, to highlight the difference between the predicted distributions.

\section{Results}

\subsection{Validation}

Four of the five decision strategies performed similarly according to the AUC statistic ( 0.52 for the Maximum Risk decision strategy) vs. 0.86-0.88 for the other four decision strategies). The Maximum Risk decision strategy performed poorly, producing a result only slightly better than chance, and is not considered further. While the differences in the AUC were marginal, the Conservative decision strategy had the highest AUC (0.88), and thus has the best average performance.

\subsection{Selecting decision strategies}

Despite the very similar AUC statistics produced for four of the five decision strategies, there are distinct differences between them at various points in ROC space (Fig. 3). For instance, the ROC curve of the Minimum Risk strategy rises quickly to a TPR of 0.75 (predicting $75 \%$ of known mesquite occurrences) whilst limiting the FPR to 0.1 . However, increasing the TPR to detect $5 \%$ more known occurrences increases the FPR to 0.35 (Fig. 3), which is less efficient than both the Conservative and the Moderate Risk strategies, as shown by their higher ROC curves over this range (Fig. 3). In comparison, the Risk Taking strategy performs worse than the three aforementioned strategies throughout this range, but is more efficient than all other strategies in the more liberal areas of ROC space, in particular, at and after $(0.4,0.92)$ (Fig. 3). Both the Minimum Risk and Risk Taking strategies were identified as potentially optimal based on the ROC convex hull (Fig. 3) and all others are not considered further.

\section{<approximate location of Fig. 3>}

\subsection{Threshold determination methods}

The TDM aimed at maximizing the OPS identified the Minimum Risk decision strategy as most optimal with an OPS of $82.5 \%$ (Table 3). Maximum efficiency was realized at a threshold of 0.67 and identified $3,074 \mathrm{~km}^{2}(2.7 \%$ of the study area) of land as having similar characteristics (Fig. 4a). This decision strategy represents the most accurate solution if it can be assumed that the validation data characterize the entire variance of the mesquite population (i.e., the population is at equilibrium). However, given that it was unable to predict $25 \%$ of the mesquite presence data (125 out of 500 known presence points) (see TPR, Table 3) the prediction may be too conservative to predict new outbreaks arising from dispersal or range expansion.

The Risk Taking strategy was the most accurate at correctly identifying $95 \%$ of the mesquite presence data (Table 3). The lowest corresponding FPR (44\%) was realized at a threshold of 0.77 , which identified $23.2 \%$ of the study area as potentially suitable. This represents an additional $23,066 \mathrm{~km}^{2}$ that was not identified by maximizing OPS (Fig. 4a). The remaining 5\% of known presence locations (false negatives) that were 
not identified by this TDM and decision strategy were all located on the peripheries of the main mesquite infestation in the Fortescue catchment (Fig. 4b). False positives were most common in the Robe catchment (64\%), compared with $21 \%$ in the Fortescue catchment and 15\% in the Eramurra catchment (Fig. 4b). Both the Robe and Eramurra catchments have been invaded relatively recently, but the Robe catchment has isolated mesquite shrubs across ca. $34 \%$ of it while mesquite is still rare in the Eramurra catchment (ca. 2\% of area surveyed) (van Klinken et al. 2007).

\section{Discussion}

\section{<approximate location of Fig. 4a-b>} $<$ colour on web and colour in print $>$

Predicting the potential distribution of invasive organisms that are not yet at equilibrium is difficult but nonetheless is critical for effective management. In general, this difficulty is borne from the assumption that absence records represent poor habitat, which may not be the case for recently introduced and actively expanding species (Hirzel et al., 2001; Elith et al., 2006). This is certainly true of the mesquite population studied here, which is approximately 70-80 years old, well within the life-span of mesquite plants, and still rapidly expanding (van Klinken et al., 2007; Robinson et al., 2008). Ideally, regular surveys should be conducted to provide information on the spread of invasive species (Hulme, 2003; Underwood et al., 2004) to ensure that absence data actually represents conditions that preclude invasion, not simply that the species has not had sufficient time to invade there. However, given rapid invasion rates, land managers require this information in advance to adopt preemptive management strategies (e.g. sufficiently extensive surveillance programs) and therefore, models adopted for management need to be able to identify these potential areas. In this paper, we have demonstrated that by incorporating different levels of risk in the decision strategy and choosing between them based on different threshold determination methods, we can expand our predictions to identify these potential areas and we can do so more accurately by comparing several strategies and eliminating sub-optimal ones that are not on the convex hull of the receiver operating characteristic (ROC) curves.

Several decision strategies fitted our validation dataset similarly well (based on the area under the curve (AUC) statistic) but produced very different predicted spatial distributions. Two of the decision strategies were considered potentially optimal based on the convex hull of the ROC curves. However, the decision strategy that assumes the population is still invading (Risk Taking strategy) predicted 8.5 times more area than the decision strategy assuming the population is at equilibrium (Minimum Risk strategy). The former decision strategy is consistent with observations of where mesquite is currently invading and posing the greatest threat within the Mardie Pastoral Lease. It identified much of the Robe catchment as being highly suitable, and much of the Eramurra catchment as being unsuitable. Although both are relatively recently invaded, invasions in the Robe catchment has been much more rapid, has already resulted in formation of dense patches, and is seen as the greatest threat by local land managers (van Klinken et al., 2007). These contrasting predictions clearly have profound consequences for designing management strategies. However, the Risk Taking decision strategy is the method of choice where omission errors (false negatives) are costly to management and commission errors (false positives) can be tolerated; although, it does require considerably more management 
resources (Lippitt et al., 2007). While model adoption is always a compromise between accuracy and costs (Store and Kangas, 2001), this combination has the most applicability for our goal of providing an early warning for detecting new outbreaks and also avoids a high level of omissions that would otherwise put containment programs at risk.

A prudent approach to ecological modeling has been to adopt several models and identify the relative advantages of each for specific management purposes (e.g. Loiselle et al., 2003; Lippitt et al., 2008) or to manipulate the weights given to criteria using sensitivity analysis (e.g. Store and Kangas, 2001). In this paper we manipulate our set of chosen weights using OWA to identify a level of risk that more directly suits the application. In our case we chose to increase risk levels to increase the number of known locations that were correctly predicted (true positives) at the cost of a larger number of false positives. Such an approach may also be beneficial for a range of studies where failure to predict potential areas is more costly than overestimation (Fielding and Bell, 1997) such as designing areas for the protection of endangered species, or searching for new populations of rare species (e.g. de Siqueira et al., 2009). We also demonstrate the ability to model populations that are at equilibrium. Alternatively, this approach could be manipulated to minimize the number of false positives so as to identify only the most suitable areas for future invasion to match resource constraints or for defining areas for species reintroduction programs (e.g. Loiselle et al., 2003). These are important considerations in the context of landscape ecological planning and hence we consider the manipulation of risk levels to suit desired purposes is likely to be a desirable quality for a range of users and applications.

Most modeling applications in landscape ecology standardize criteria into binary responses (crisp standardization) using presence-only data (e.g. profile models). These types of models remain popular (e.g. Tsoar et al., 2007), primarily due to the historical availability and ease of collection of presence-only information. However, unlike the standardization procedure used in this study, crisp standardization does not incorporate the notion that some conditions are more favorable than others and the differences are continuous (Heuvelink and Burrough, 1993) and yet continuous standardization using fuzzy membership functions (FMFs) does not require any more data (e.g. absence locations) than that used for profile models, for example. While FMFs are not new, the search for improved methods for predicting species distributions from presence-only data is currently topical (e.g. Elith et al., 2006) and Robertson et al. (2004) have shown FMFs have the ability to improve model accuracy over crisp standardization. Another attractive feature of continuous standardization, as shown in this study, is that it allows favorable criteria to compensate (trade-off) for less favorable criteria, which is not possible with crisp standardization where all criteria need to be 1 for the model to return a positive result.

The AUC statistic obtained from ROC analysis is a current standard practice for assessing, comparing and selecting between different models or decision strategies (Zweig and Campbell, 1993; Austin, 2007). This is primarily because it avoids the subjectivity in selecting one particular threshold by summarizing the overall model performance over all possible thresholds (Fielding and Bell, 1997). However, the AUC has recently been scrutinized for its inability to be used as a comparative measure of accuracy (Termansen et al., 2006; Elith et al., 2006; Austin, 2007; Lobo et 
al., 2008; Peterson et al., 2008). In this study we found very similar AUC statistics between four of the five decision strategies implemented but obvious differences in their corresponding ROC curves and thus very different patterns of predicted suitability. Despite the similar AUC statistics only two of the five decision strategies fitted our goals. The decision strategy with the highest AUC (Conservative) was unable to achieve either of these goals. Therefore, the most attractive feature of ROC analysis appears to be the ability to examine the true and false positive rates over all thresholds to assist selection of the most appropriate decision strategy, according to the user's goals (Jiménez-Valverde and Lobo, 2007; Lobo et al., 2008). Based on our results and those of others (e.g. Termansen et al., 2006) use of the AUC statistic for any other purpose than to summarize the performance over all thresholds should be discouraged.

\section{Conclusions}

Invasive species distribution modeling is challenging as these species have rarely reached equilibrium within their environment. Therefore, absence records may not represent habitat that is unsuitable, but rather habitat that has yet to be invaded. This research compared several different decision strategies that were developed by coupling ordered weighted averaging to a multi-criteria evaluation model. Based on the area under the curve obtained from receiver operating characteristic (ROC) analyses, four out of five models could not be separated. However, the decision strategies showed different patterns in ROC space and sub-optimal strategies could be selected based on the convex hull of the ROC curves. Threshold determination methods could be used to further explore the applicability of the remaining strategies for management purposes. We found that adopting the most statistically accurate decision strategy, which was also the decision strategy assuming the population was at equilibrium, would vastly underestimate the area requiring surveillance for the invasive mesquite population under study. Instead, a decision strategy that was potentially optimal in the more liberal areas of ROC space was preferable for our management purposes. The ability to test multiple decision strategies was found to be extremely valuable for our purposes and can be readily adapted to applications requiring similar flexibility. For example, higher levels of risk may be more desirable for designing areas for the protection of endangered species, or searching for new populations of rare species (e.g. de Siqueira et al., 2009). In contrast, the tools used here can also be used for modeling species at equilibrium (as shown) or to minimize risk for applications such as species reintroduction programs.

\section{Acknowledgements}

This research was supported under Australian Research Council's ARC-Linkage funding scheme (Project Number: LP0454890). The views expressed in this paper are those of the authors and are not necessarily those of the Australian Research Council. We would like to thank the Pilbara Mesquite Management Committee (PMMC) and, in particular, Linda Anderson from the PMMC for field assistance and logistical support. Additionally, we would like to thank Damian Shepherd from the Department of Agriculture and Food, Western Australia (DAFWA), for assistance with GIS datasets, the managers of Mardie Station (Richard and Lindy Climas) for logistical support, and Dr. Justine Murray (CSIRO), Dr. Paul Novelly (DAFWA) and Dr. Tom Schut (Curtin University) for comments on a draft manuscript. Lastly, we thank the two anonymous reviewers for their suggested improvements and additions. 
References

Archer, S., 1995. Tree-grass dynamics in a Prosopis-thornscrub savanna parkland: reconstructing the past and predicting the future. Ecosci. 2, 83-99.

Austin, M.P., 2002. Spatial prediction of species distribution: an interface between ecological theory and statistical modelling. Ecol. Model. 157, 101-118.

Austin, M., 2007. Species distribution models and ecological theory: a critical assessment and some possible new approaches. Ecol. Model. 200, 1-19.

Ayalew, L., Yamagishi, H., 2005. The application of GIS-based logistic regression for landslide susceptibility mapping in the Kakuda-Yahiko Mountains, Central Japan. Geomorphol. 65, 15-31.

Beaumont, L.J., Gallagher, R.V., Thuiller, W., Downey, P.O., Leishman, M.R., Hughes, L., 2009. Different climatic envelopes among invasive populations may lead to overestimations of current and future biological invasions. Divers. Distrib. 15, 409-420.

Beerling, D.J., Huntley, B., Bailey, J.P., 1995. Climate and the distribution of Fallopia japonica: use of an introduced species to test the predictive capacity of response surfaces. J. Veg. Sci. 6, 269-282.

Brown, J.R., Archer, S., 1987. Woody plant seed dispersal and gap formation in a North-American sub-tropical savanna woodland: the role of domestic herbivores. Vegetatio. 73, 73-80.

Burrough, P.A., McDonnell, R.A., 1998. Principles of Geographical Information Systems: Spatial Information Systems and Geostatistics, Oxford University Press, Oxford.

Cantor, S.B., Sun, C.C., Tortolero-Luna, G., Richards-Kortum, R., Follen, M., 1999. Comparison of $\mathrm{C} / \mathrm{B}$ ratios from studies using receiver operating characteristic curve analysis. J. Clin. Epidemiol. 52, 885-892.

De Meyer, M., Robertson, M.P., Peterson, A.T., Mansell, M.W., 2007. Ecological modeling and potential geographic distributions of Mediterranean fruit fly (Ceratitis capitata) and Natal fruit fly (Ceratitis rosa). J. Biogeogr. 35, 270-281.

de Siqueira, M.F., Durigan, G., de Marco Júnior, P., Peterson, A.T., 2009. Something from nothing: using landscape similarity and ecological niche modeling to find rare plant species. J. Nat. Conserv. 17, 25-32.

Eastman, J.R., Kyem, P.A.K., Toledano, J., Jin, W., 1993. Explorations in geographic systems technology, vol. 4, GIS and Decision Making, Geneva, Switzerland, UNITAR.

Elith, J., Graham, C.H., Anderson, R.P., Dudik, M., Ferrier, S., Guisan, A., Hijmans, R. J., Huettmann, F., Leathwick, J.R., Lehmann, A., Li, J., Lohmann, L.G., Loisell, B.A., Manion, G., Moritz, C., Nakamura, M., Nakazawa, Y., Overton, 
J., Peterson. A.T., Phillips, J., Richardson, K., Scahetti-Pereira, R., Schapire, R.E., Soberon, J., Williams, S., Wisz, M.S., Zimmermann, N. E., 2006. Novel methods to improve prediction of species' distributions from occurrence data. Ecography 29, 129-151.

Fawcett, T., 2006. An introduction to ROC analysis. Pattern Recognit. Lett. 27, 861874.

Fielding, A.H., Bell, J.F., 1997. A review of methods for the assessment of prediction errors in conservation presence/absence models. Environ. Conserv. 24, 38-49.

Franklin, J., 1995. Predictive vegetation mapping: geographic modelling of biospatial patterns in relation to environmental gradients. Prog. Phys. Geog. 19, 474-499.

Gessler, P.E., Moore, I.D., McKenzie N.J., Ryan, P.J., 1995. Soil-landscape modelling and spatial prediction of soil attributes. Int. J. GIS 9, 421-432.

Gibbens, R.P., Beck, R.F., McNeely, R.P., Herbel, C.H., 1992. Recent rates of mesquite establishment in the Northern Chihuahan Desert. J. Range Manage. 45, 585-588.

Goslee, S.C., Havstad, K.M., Peters, D.P.C., Rango, A., Schlesinger, W.H., 2003. High-resolution images reveal rate and pattern of shrub encroachment over six decades in New Mexico, U.S.A. J. Arid Environ. 54, 755-767.

Guisan, A., Zimmermann, N.E., 2000. Predictive habitat distribution models in ecology. Ecol. Model. 135, 147-186.

Hennessy, J.T., Gibbens, R.P., Tromble, J.M., Cardenas, M., 1983. Vegetation changes from 1935 to 1980 in mesquite dunelands and former grasslands of southern New Mexico. J. Range Manage. 36, 370-374.

Heuvelink, G.B.M., Burrough, P.A. 1993. Error propagation in cartographic modelling using Boolean logic and continuous classification. Int. J. Geogr. Inf. Sci. 7, 231-246.

Hirzel, A.H., Helfer, V., Metral, F., 2001. Assessing habitat suitability models with a virtual species. Ecol. Model. 145, 111-121.

Hulme, P.E., 2003. Biological invasions: winning the science but losing the conservation war? Oryx 37, 178-193.

Jiang, H. \& Eastman, J.R., 2000. Application of fuzzy measures in multi-criteria evaluation in GIS. Int. J. Geogr. Inf. Sci. 14, 173-184.

Jiménez-Valverde, A., Lobo, L.M., 2007. Threshold criteria for conversion of probability of species presence to either-or presence-absence. Acta Oecologica 31, 361-369. 
Jiménez-Valverde, A., Lobo, L.M., Hortal, J., 2008. Not as good as they seem: the importance of concepts in species distribution modeling. Divers. Distrib. 14, 885-890.

Lippitt, C.D., Rogan, J., Toledano, J., Sangermano, F., Eastman, J.R., Mastro, V. Sawyer, A., 2008. Incorporating anthropogenic variables into a species distribution model to map gypsy moth risk. Ecol. Model. 210, 339-350.

Liu, C, Berry, P.M., Dawson, T.P., Pearson, R.G., 2005. Selecting thresholds of occurrence in the prediction of species distributions. Ecography 28, 385-393.

Lobo, J.M., Jiménez-Valverde, A., Real, R., 2008. AUC: a misleading measure of the performance of predictive distribution models. Glob. Ecol. Biogeogr. 17, 145151.

Loiselle, B.A., Howell, C.A., Graham, C.H., Goerck, J.M., Brooks, T., Smith, K.G.. Williams, P.H., 2003. Avoiding pitfalls of using species distribution models in conservation planning. Conserv. Biol. 17, 1591-1600.

Malczewski, J., 1999. GIS and mutlicriteria decision analysis. John Wiley and Sons, Brisbane.

Malczewski, J., 2006. Ordered weighted averaging with fuzzy quantifiers: GIS-based multicriteria evaluation for land-use suitability analysis. Int. J. Appl. Earth Obs. Geoinf. 8, 270-277.

Manel, S., Williams, H.C., Ormerod, S.J., 2001. Evaluating presence-absence models in ecology: the need to account for prevalence. J. Appl. Ecol. 38, 921-931.

Meadly, G.R.W., 1962. Weeds of Western Australia - mesquite. J. Agric. WA 3, 729739.

Mgidi, T.N., Le Maitre, D.C., Schonegevel, L., Nel, J.L., Rouget, M., Richardson, D.M., 2007. Alien plant invasions - incorporating emerging invaders in regional prioritization: a pragmatic approach for Southern Africa. J Environ. Manage. 84, 173-187.

Morisette, J.T., Jarnevich, C.S., Ullah, A., Cai, W., Pedelty, J.A., Gentle, J.E., Stohlgren, T.J., Schnase, J.L., 2006. A tamarisk habitat suitability map for the continental United States, Front. Ecol. Environ. 4, 11-17.

Olivera, F., Furnans, J. Maidment, D., Djokic, D., Ye, Z., 2002. Drainage Systems in: ArcHydro: GIS for Water resources. (ed. by D. Maidment), pp. 55-86, ESRI Press, Redlands, California.

Pasiecznik, N.M., Felker, P., Harris, P.J.C., Harsh, L.N., Cruz, G., Tewari, J.C., Cadoret, K., Maldonado, L.J., 2001. The Prosopis juliflora - Prosopis pallida complex: a monograph, HDRA Coventry, UK. 
Payne, A.L., Mitchell, A.A., 2002. Pastoral condition guides for the Pilbara. Miscellaneous Publication 19/2002, Department of Agriculture, Perth, Australia.

Peterson, A.T., Papes, M., Soberón, J., 2008. Rethinking receiver operating characteristic analysis applications in ecological niche modeling, Ecol. Model. 213, 63-72.

Pontius, R.G. Jr., Schneider, L.C., 2001. Land-cover change model validation by an ROC method for the Ipswich watershed, Massachusetts, USA, Agric. Ecosyst. Environ. 85, 239-248.

Rejmánek, M., Pitcairn, M.J., 2002. When is eradication of exotic pest plants a realistic goal? Turning the tide: the eradication of invasive species (ed. by C.R. Veitch and M.N. Clout), pp. 249-252, IUCN SSC Invasive Species Specialist Group, Gland, Switzerland, and Cambridge, UK.

Robertson, M.P., Peter, C.I., Villet, M.H., Ripley, B.S., 2003. Comparing models for predicting species' potential distributions: a case study using correlative and mechanistic predictive modelling techniques. Ecol. Model. 164, 153-167.

Robertson, M.P., Villet, M.H., Palmer, A.R., 2004. A fuzzy classification technique for predicting species' distributions: applications using invasive alien plants and indigenous insects. Divers. Distrib. 10, 461-474.

Robinson, T.P., van Klinken, R.D., Metternicht, G.I., 2008. Spatial and temporal rates and patterns of mesquite (Prosopis spp.) invasion in Western Australia. J. Arid Environ. 72, 175-188.

Saaty, R.W., 1987. The analytic hierarchy process - what it is and how it is used. Math. Model. 9, 161-176.

Saaty, T.L., 1977. A scaling method for priorities in hierarchical structures. J. Math. Psychol. 15, 234-281.

Store, R., Kangas, J., 2001. Integrating spatial multi-criteria evaluation and expert knowledge for GIS-based habitat suitability modelling. Landsc. Urban Plan. 55, 79-93.

Sutherst, R.W., Bourne, A.S., 2009. Modelling non-equilibrium distributions of invasive species: a tale of two modelling paradigms. Biol. Invasions 11, 12311237.

Termansen, M., McClean, C.J., Preston, C.D., 2006. The use of genetic algorithms and Bayesian classification to model species distributions. Ecol. Model. 192, 410-424.

Thorp, J.R., Lynch, R., 2000. The determination of weeds of national significance, National Weeds Strategy Executive Committee, Launceston. 
Tsoar, A., Allouche, O., Steinitz, O., Rotem, D., Kadmon, R., 2007. A comparative study of presence-only methods for modelling species distribution. Divers.

Distrib. 13, 387-405.

Underwood, E.C., Klinger, R., Moore, P.E., 2004. Predicting patterns of non-native plant invasions in Yosemite National Park, California, USA, Divers. Distrib. 10, 447-459.

Václavík, T., Meentemeyer, R.K., 2009. Invasive species distribution modeling (iSDM): are absence data and dispersal constraints needed to predict actual distributions? Ecol. Model. 220, 3248-3258.

van Klinken, R.D., Graham, J., Flack, L.K., 2006. Population ecology of hybrid mesquite (Prosopis species) in Western Australia: how does it differ from native range invasions and what are the implications for impacts and management? Biol. Invasions 8, 727-741.

van Klinken, R.D., Shepherd, D., Parr, R., Robinson, T.P., Anderson, L., 2007. Mapping mesquite (Prosopis) distribution and density using visual aerial surveys. Range. Ecol. Manage. 60, 408-416.

van Klinken, R.D., Campbell, S., 2009. Australian weeds series: Prosopis species. Australian Weeds Series, Volume 3 (ed. by F.D. Panetta), pp. 238-273, R.G. and F.J. Richardson, Meredith, Victoria, Australia,

van Klinken, R.D., Lawson, B.E., Zalucki, M.P., 2009. Predicting invasions in Australia by a Neotropical shrub under climate change: the challenge of novel climates and parameter estimation. Glob. Ecol. Biogeogr. 18, 688-700.

van Vreeswyk, A.M.E, Payne, A. L., Leighton, K. A., 2004. Pastoral resources and their management in the Pilbara Region of Western Australia, Department of Agriculture, Miscellaneous Publication 21/2004.

Yager, R.R., 1988. On ordered weighted averaging aggregation operators in multicriteria decision making. IEEE Trans. Sys. Man Cybern. 18, 183-190.

Zadeh, L.A., 1965. Fuzzy sets. Inform. Control 8, 338-353.

Zweig, M.H., Campbell, G., 1993. Receiver-operating characteristic (ROC) plots: a fundamental evaluation tool in clinical medicine. Clin. Chem. 39, 561-577. 


\section{List of Tables}

Table 1 Standardized scores developed for the land use and pastoral potential criteria used in the multi-criteria evaluation (MCE) model

Table 2 Pair-wise comparison matrix and derived weights for each criterion used for determining the potential distribution of mesquite. The pair-wise comparison matrix was developed by comparing each criterion against the other criteria on the 9-point scale. Higher values suggest the row criterion is more important than the column criterion that it is compared to. Any rating at position $i, j$ is the reciprocal of that in position $j, i$. By definition the diagonal entries are always equal to 1 . The Principal Eigenvector of the matrix yields the criterion weights. The consistency ratio of the matrix is 0.01 (see text).

Table 3 Comparison of accuracy statistics obtained from alternative threshold determination methods on the two decision strategies that were on the convex hull of ROC space.

\section{List of Figures}

Figure 1 Fuzzy membership function for standardizing the compound topographic index. Points of inflection $(a=5.5, b=9.4)$ are the control points used to construct the curve (see text).

Figure 2 The decision strategy space triangle (after Jiang and Eastman, 2000) depicting different levels of risk and trade-off of the five decision strategies implemented. Values inside square brackets indicate the order weights and coefficients of risk and trade-off are shown in round brackets, respectively.

Figure 3 Receiver Operating Characteristic (ROC) curves of the five decision strategies assessed. The grey background represents the ROC convex hull. Curves are obscured where the true positive rate (TPR) and false positive rate (FPR) coordinates are identical.

Figure 4 a) Map showing the two different threshold determination methods (true positive rate $(\mathrm{TPR})=95 \%$ and Maximum overall prediction success (OPS) applied to the two decision strategies (Risk Taking and Minimum Risk, respectively). The marginal class includes all other areas that were not identified from either decision strategy; b) Map showing the spatial arrangement of the validation data classified into correct predictions (true positives/negatives) and omission (false negative) and commission (false positives) errors based on the Risk Taking decision strategy (TPR =95\%). 
1

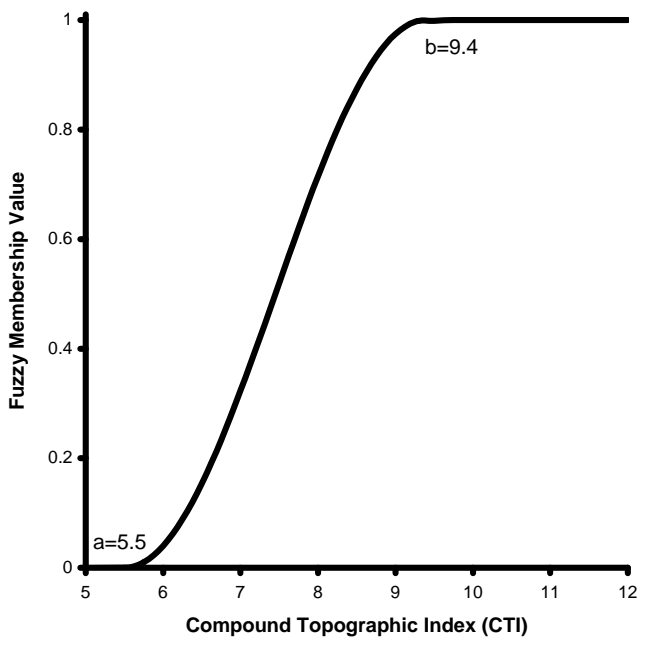

3

4

5

6

7

8

9

10

11

12

13

14

15

16

17

18

19

20

21

22

23

24

25

26

27

28

29

30

31

32

33

34

35

36

37 
33

34

35

36

37

38

39

40

41

42

43

44

45

46

47

48

49

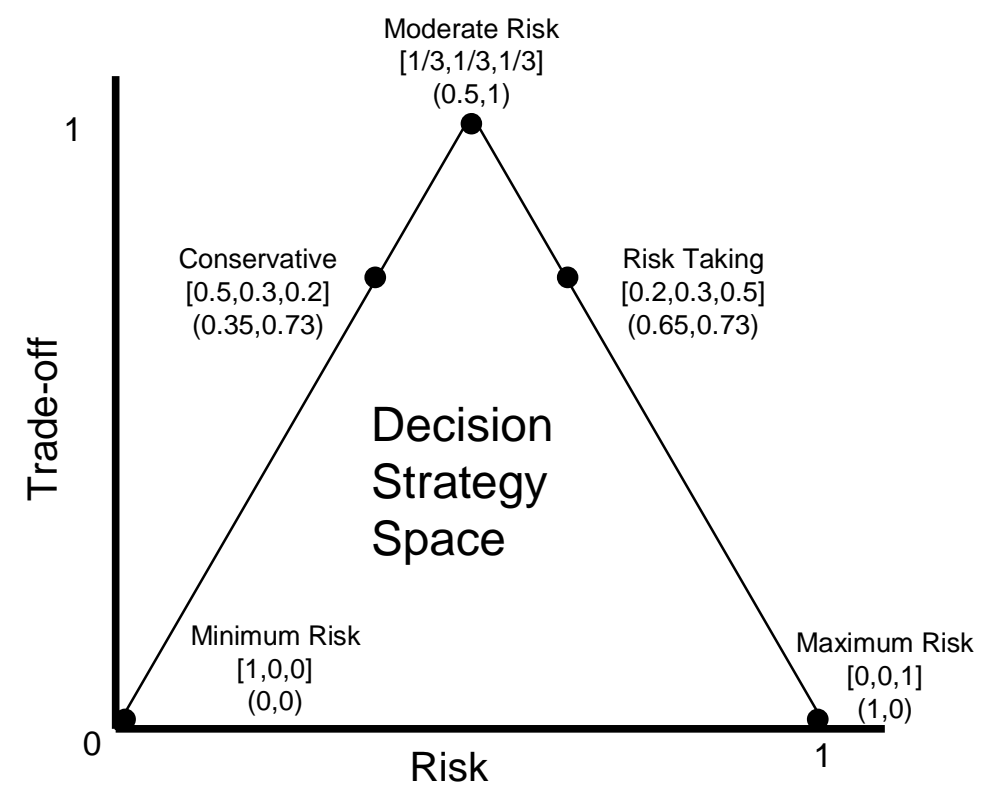

50 
1

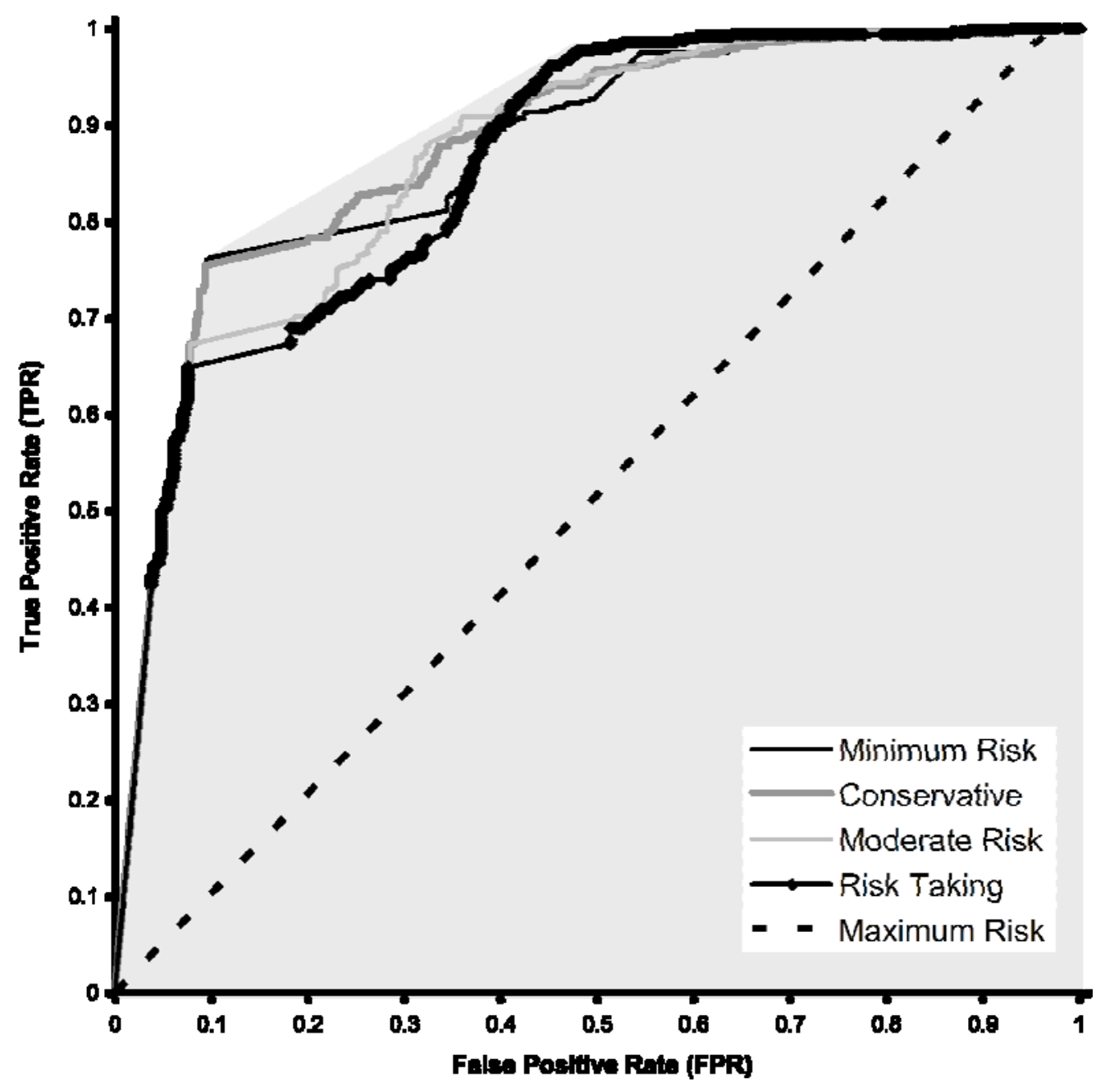

3

4

5

6 


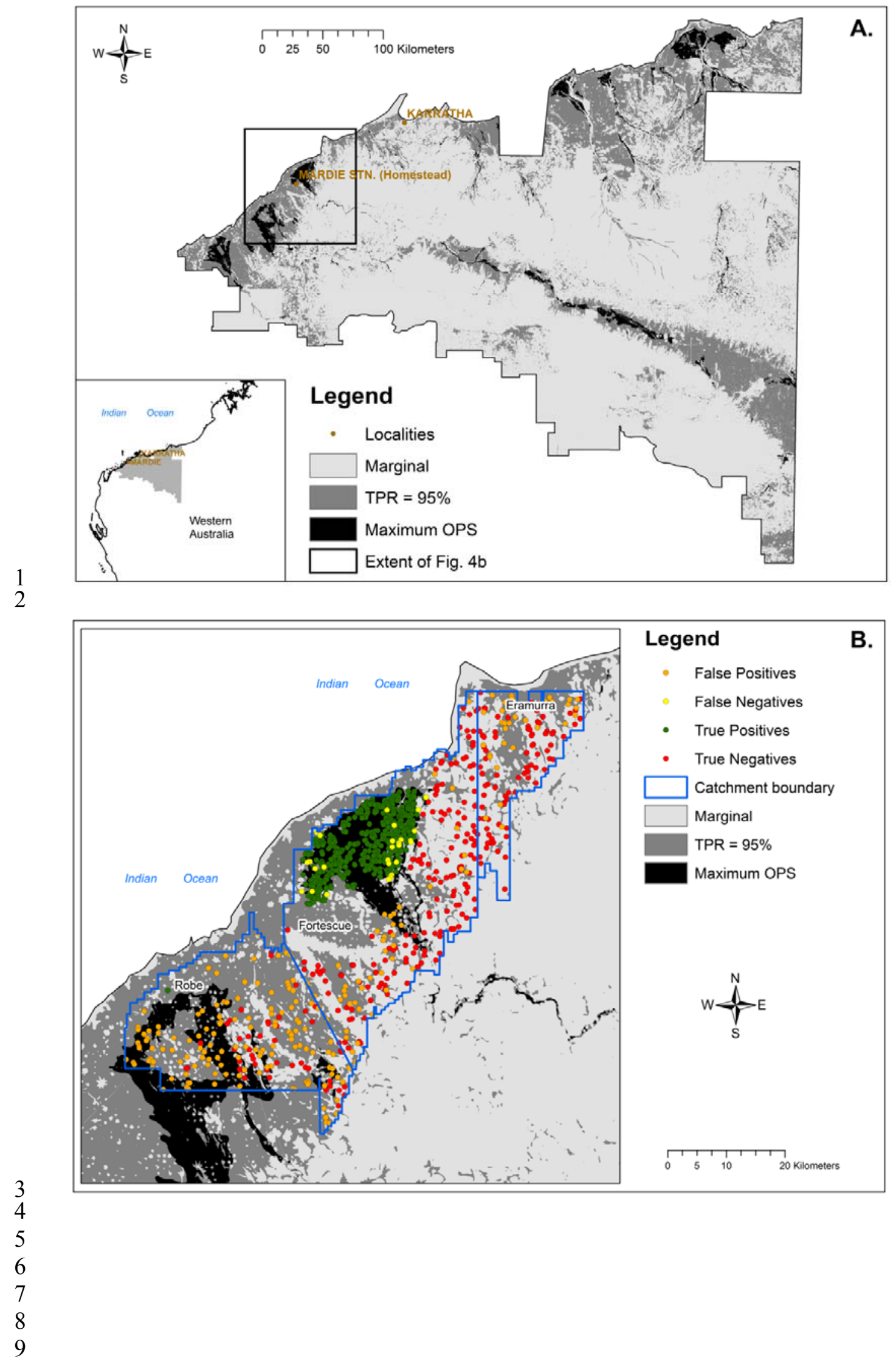


1

\begin{tabular}{lll}
\hline Criterion & Class & Standardized Score \\
\hline Pastoral Potential & Very high & 1 \\
& High & 0.64 \\
& Moderate & 0.36 \\
& Low & 0.16 \\
& Very low & 0.04 \\
\hline Land Use & Livestock grazing (cattle) & 1 \\
& Managed resource protection & 0.25 \\
& Minimum intervention use & 0.25 \\
& Species management area & 0.25 \\
& Traditional indigenous use & 0.25 \\
& National park & 0.25 \\
Strict nature reserves & 0.25 \\
\hline
\end{tabular}

2

3

4

5

6

7

8

9 


\begin{tabular}{lllll}
\hline & CTI & Pastoral potential & Land use & Weight \\
\hline CTI & 1 & 2 & 3 & 0.54 \\
Pastoral potential & $1 / 2$ & 1 & 2 & 0.30 \\
Land use & $1 / 3$ & $1 / 2$ & 1 & 0.16 \\
\hline
\end{tabular}

1

3

4

5

6

7

8

9

10

11

12

13

14

15

16

17

18

19

20

21

22

23

24

25

26

27

28

29

30

31

32

33

34

35

36

37

38

39

40

41

42

43

44

45

46

47 


\begin{tabular}{|l|l|l|l|l|l|l|l|}
\hline $\begin{array}{l}\text { Threshold } \\
\text { Determination } \\
\text { Method }\end{array}$ & $\begin{array}{l}\text { Decision } \\
\text { Strategy }\end{array}$ & $\begin{array}{l}\mathrm{TPR}^{1} \\
(\%)\end{array}$ & $\begin{array}{l}\mathrm{FPR}^{2} \\
(\%)\end{array}$ & OPS (\%) & $\begin{array}{l}\text { ME } \\
\text { Statistic } \\
(\%)^{4}\end{array}$ & $\begin{array}{l}\text { Threshold } \\
\text { value }\end{array}$ & $\begin{array}{l}\text { Area } \\
\text { predicted } \\
\left(\mathrm{km}^{2}\right)^{5}\end{array}$ \\
\hline Maximize OPS & Minimum Risk & 75 & 10 & 82.5 & 65 & $\geq 0.67$ & 3074 \\
\hline & Risk Taking & 65 & 8 & 78.8 & 57 & $\geq 0.92$ & 13,141 \\
\hline TPR $=95 \%$ & Minimum Risk & 95 & 54 & 70.5 & 41 & $\geq 0.25$ & 13,122 \\
\hline & Risk Taking & 95 & 44 & 80.5 & 51 & $\geq 0.77$ & 26,140 \\
\hline
\end{tabular}

${ }^{1}$ True positive rate (TPR) is the number of known presence locations that were identified by the decision strategy, divided by the total number of known presence locations in the validation data set.

${ }^{2}$ False positive rate (FPR) is the number of known absence points that were identified by the decision strategy to be suitable

divided by the total number of known absence locations in the validation data set.

${ }^{3}$ Overall Prediction Success (OPS) is the sum of the number of correctly identified presence locations to the number of correctly

identified absence locations divided by the number of points in the validation dataset (1000), expressed as a percentage.

${ }^{4}$ The Maximum efficiency (ME) statistic maximizes the difference between the TPR and the FPR.

${ }^{5}$ The total area identified as suitable for each decision strategy based on the threshold value. 